\title{
High Surface Area of Porous Silicon Drives Desorption of Intact Molecules
}

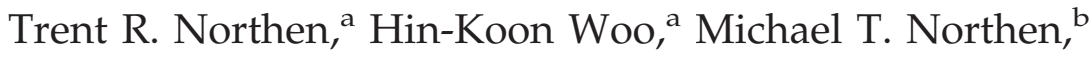

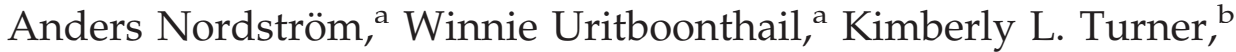 \\ and Gary Siuzdak ${ }^{a}$ \\ ${ }^{a}$ Center for Mass Spectrometry, The Scripps Research Institute, La Jolla, California, USA \\ ${ }^{b}$ Department of Mechanical Engineering, University of California at Santa Barbara, Santa Barbara, \\ California, USA
}

\begin{abstract}
The surface structure of porous silicon used in desorption/ionization on porous silicon (DIOS) mass analysis is known to play a primary role in the desorption/ionization (D/I) process. In this study, mass spectrometry and scanning electron microscopy (SEM) are used to examine the correlation between intact ion generation with surface ablation and surface morphology. The DIOS process is found to be highly laser energy dependent and correlates directly with the appearance of surface ions $\left(\mathrm{Si}_{n}^{+}\right.$and $\left.\mathrm{OSiH}^{+}\right)$. A threshold laser energy for DIOS is observed (10 $\left.\mathrm{mJ} / \mathrm{cm}^{2}\right)$, which supports that DIOS is driven by surface restructuring and is not a strictly thermal process. In addition, three DIOS regimes are observed that correspond to surface restructuring and melting. These results suggest that higher surface area silicon substrates may enhance DIOS performance. A recent example that fits into this mechanism is the surface of silicon nanowires, which has a high surface energy and concomitantly requires lower laser energy for analyte desorption. (J Am Soc Mass Spectrom 2007, 18, 1945-1949) (c) 2007 American Society for Mass Spectrometry
\end{abstract}

$\mathrm{D}$ esorption/ionization on porous silicon (DIOS) [1] mass analysis is fundamentally different from other surface-based desorption/ionization (D/I) approaches [2-4] because it is largely dependent on the silicon surface structure and does not require a matrix. Other surfaces have also been shown to generate intact molecular ions into the gas phase, including GaAs and GaN [5], immobilized carbon nanotubes [6], silicon nanowires $[7,8]$, and lithographically patterned and roughened silicon surfaces [9]. In particular, the porous silicon surface has demonstrated unique performance capabilities, allowing the analysis of a wide range of molecules [10] and peptide analysis at the 800 yoctomole level [11]. Given its novel means of generating ions, a more complete understanding on how these ions are transferred intact into the gas phase has practical as well as fundamental scientific implications for surface-based mass analysis.

The high surface area of the porous silicon surface is critical to DIOS performance [5, 12]. Porous silicon can have a surface area a million times greater than that of a planar surface $\left(>100 \mathrm{~m}^{2} / \mathrm{cm}^{2}\right.$ of etched material). This high surface area significantly lowers the melting point of silicon; pore coalescence and structural collapse were observed at $900{ }^{\circ} \mathrm{C}[13]$, whereas bulk silicon melting

Address reprint requests to Dr. Gary Siuzdak, Center for Mass Spectrometry, The Scripps Research Institute, 10550 North Torrey Pines Road, BCC-007, La Jolla, CA 92037. E-mail: siuzdak@scripps.edu occurs at $1410{ }^{\circ} \mathrm{C}[14]$. The driving force for this melting process is a reduction in surface energy of the porous silicon $\left(0.2 \mathrm{~J} / \mathrm{cm}^{2}\right.$ for porous silicon versus $0.0001 \mathrm{~J} / \mathrm{cm}^{2}$ planar silicon) [13]. In fact, the typical nitrogen laser energy $\left(15 \mathrm{~mJ} / \mathrm{cm}^{2}\right.$-pulse) commonly used in DIOS is known to induce melting of porous silicon [15]. The laser surface heating and internal energy transfer are estimated to result in surface temperatures as high as $600 \mathrm{~K}$ [16]. Another observation supporting this idea is that lower laser intensities are required when DIOS is performed on silicon nanowires $[7,8,16]$, due to the extremely high surface area.

It is also important to note that the porous surface may serve as a proton source. Although it has been suggested that solvent or residual hydrofluoric acid (HF) provides sources of protons for DIOS [16], surface heating [17] and photochemical processes [18, 19] are known to causes loss of surface hydrogen from porous silicon. The hydrogen-rich surface $\mathrm{SiH}_{x}(\mathrm{x}=1-3)$ [20] is a result of the anodic etching process in HF used to make the porous silicon. Although it is not clear whether hydrogen is lost as protons, hydrogen loss from the porous silicon surface does not fit a secondorder rate equation, suggesting loss is not strictly as molecular hydrogen [15]. In addition, the strong electric fields used in DIOS $(25 \mathrm{kV})$ may enhance proton formation where the surface acts as an electron sink.

In this study, we examine the process of laserinduced surface restructuring as a driver for analyte 
desorption in DIOS. The surface area reduction could be in the form of surface melting and surface area rearrangement. The roles of solvent and residual $\mathrm{HF}$ in the $\mathrm{D} / \mathrm{I}$ process are also investigated.

\section{Experimental}

\section{Preparation of Porous Silicon Surfaces}

Hydrofluoric acid (HF) and methanol were purchased from Fisher Scientific (Fair Lawn, NJ, USA) with the highest purity available. A detailed description of the preparation of DIOS surfaces is reported elsewhere [21]. Briefly, single-side polished, low-resistivity (0.01-0.02 $\Omega$-cm) N-type $\langle 100\rangle 500-550 \mu \mathrm{m}$ thick silicon wafers were obtained from Silicon Quest International (Santa Clara, CA, USA) and cut into $3.3 \times 3.3 \mathrm{~cm}$ pieces. These were then washed with methanol and blown dry with nitrogen. Etching was performed by clamping chips between a gold foil (anode) in a Teflon chamber, which was subsequently filled with $25 \%$ ethanolic HF solution. Illumination of the polished (top) side of the chip was accomplished using a model I-250 $250 \mathrm{~W}$ quartzhalogen lamp (CUDA Fiberoptics, Jacksonville, FL, USA) filtered through a 100 spot mask. A platinum loop immersed in the HF solution served as the cathode. Etchings were performed in constant-current mode (5 $\mathrm{mA}$ ) for 3 min using a Bio-Rad PowerPack1000 power supply (Hercules, CA, USA).

\section{Surface Treatments}

Etched porous silicon surfaces were rinsed with methanol and blown dry with nitrogen. Oxidized surfaces were prepared from the freshly etched porous silicon surfaces for $30 \mathrm{~s}$ with a stream $(0.5 \mathrm{~g} / \mathrm{h})$ of ozone produced from a Expotech (Houston, TX, USA) ozone generator. All surfaces were baked at $150{ }^{\circ} \mathrm{C}$ in a VWR $1430 \mathrm{D}$ vacuum oven (West Chester, PA, USA) using a Pfeiffer UNO 030B rotary pump (Nashua, NH, USA). After the vacuum baking, DIOS plates were cut into two pieces-one for vapor deposition and one for solvent deposition. Amines 1-tetradecylamine, 1hexadecylamine, and octadecylamine (Aldrich, St. Louis, MO, USA) were vapor deposited onto the various surface treatments at the same time using a custommade atmospheric pressure desposition chamber. DIOS chips were suspended at the top of a glass chamber and were rotated above a mixture of amines heated to about $100^{\circ} \mathrm{C}$, allowing the amine vapor to directly deposit onto the DIOS surface. The deposition time was about 30 s. Unfortunately, the current deposition setup did not allow a fine control on the amount of analyte deposited.

\section{Mass Spectrometry and Surface Characterization}

The DIOS approach uses a matrix-assisted laser desorption/ionization (MALDI) time-of-flight mass spectrometer where ions are observed as a result of nanosecond pulsed laser irradiation at $337 \mathrm{~nm}$ (pulse width $=4 \mathrm{~ns}$ ). Porous silicon chips were mounted to modified MALDI targets and mass spectra were collected using a Voyageur DE-STR (Applied Biosystems, Foster City, CA, USA). Data collection was performed with one laser shot per spot, ten shots per area, and five replicates per treatment. SEM images were collected on native ( $\mathrm{SiH})$ surfaces prepared as described using a FEI Quanta FEG 200F Scanning Electron Microscope (Hillsboro, OR, USA). Estimates of surface area reduction are based on SEM image analysis comparing the area of reflected electrons (bright areas) versus the total surface area. Time-of-flight secondary ion mass spectrometry (TOF-SIMS) was performed on native porous silicon surface using Physical Electronics TRIFT III (Chanhassen, MN, USA) with FEI Gold Ion Gun (Hillsboro, OR, USA).

\section{Results and Discussion}

\section{Solvent Effects}

The solvent has been suggested to promote the D/I of ions from porous silicon. In separate studies, the surface wetting properties of the solvent were previously demonstrated to play a role in D/I process [5]. In addition, the solvent and/or residual HF [16] may also serve as a proton source for ionization. It was therefore important to examine these solvent effects. To minimize the solvent effect, vacuum baking the DIOS surface and vapor depositing the analyte were used to minimize residual solvent on the surface. 1-Hexadecylamine was vaporand solvent-deposited onto the same DIOS chip in different spots. The concentration of solvent deposition was $1 \mathrm{nmol} / \mu \mathrm{L}$ in methanol. Although this approach may not completely remove all solvent, if solvent or residual $\mathrm{HF}$ were critical to the $\mathrm{D} / \mathrm{I}$ process, a strong reduction in DIOS signal would be expected. However, as shown in Figure 1a, no dramatic change in DIOS performance was observed between solvent- and vapor-deposited analyte, supporting the view that the surface itself is a primary proton source. This is consistent with the observation that silicon nanowires produced without HF [7] also have DIOS activity. Moreover, high ion intensity was observed with vapordeposited analyte, suggesting that surface wetting is not absolutely essential for the production of DIOS signal.

\section{Laser Intensity, Surface Restructuring, and DIOS Activity}

The laser-induced surface rearrangement of DIOS surfaces was examined using SEM imaging to measure surface morphological changes at different laser intensities. SEM images taken from single laser shot are shown in Figure 2. Initially, the DIOS surface is characterized as a porous surface with $20-100 \mathrm{~nm}$ pore size 
(Figure 2a; no laser irradiation). Similar to the reported literature [13], surface melting was observed upon laser irradiation: one pulse of $15 \mathrm{~mJ} / \mathrm{cm}^{2}$-pulse results in significant surface restructuring (Figure $2 b$ ) as characterized by an increase in surface roughness and pore size. The estimated surface area reduction is roughly $30 \%$ relative to the original DIOS surface by measuring the porous density in the SEM images. Irradiation with $110 \mathrm{~mJ} / \mathrm{cm}^{2}$-pulse results in pore coalescence and the formation of what appear to be spherical structures (Figure $2 c)$ with essentially a loss in surface area $(\sim 55 \%$ reduction relative to Figure 2a). Given this surface area loss, it is expected that adsorbed materials (e.g., analyte of interest, hydrogen, and surface oxide) must desorb or ablate as a result of surface heating and restructuring. At extremely high laser power (Figure $2 \mathrm{~d}, 970 \mathrm{~mJ} / \mathrm{cm}^{2}$ ), pores are melted and fused together with a complete loss of porosity (surface area).

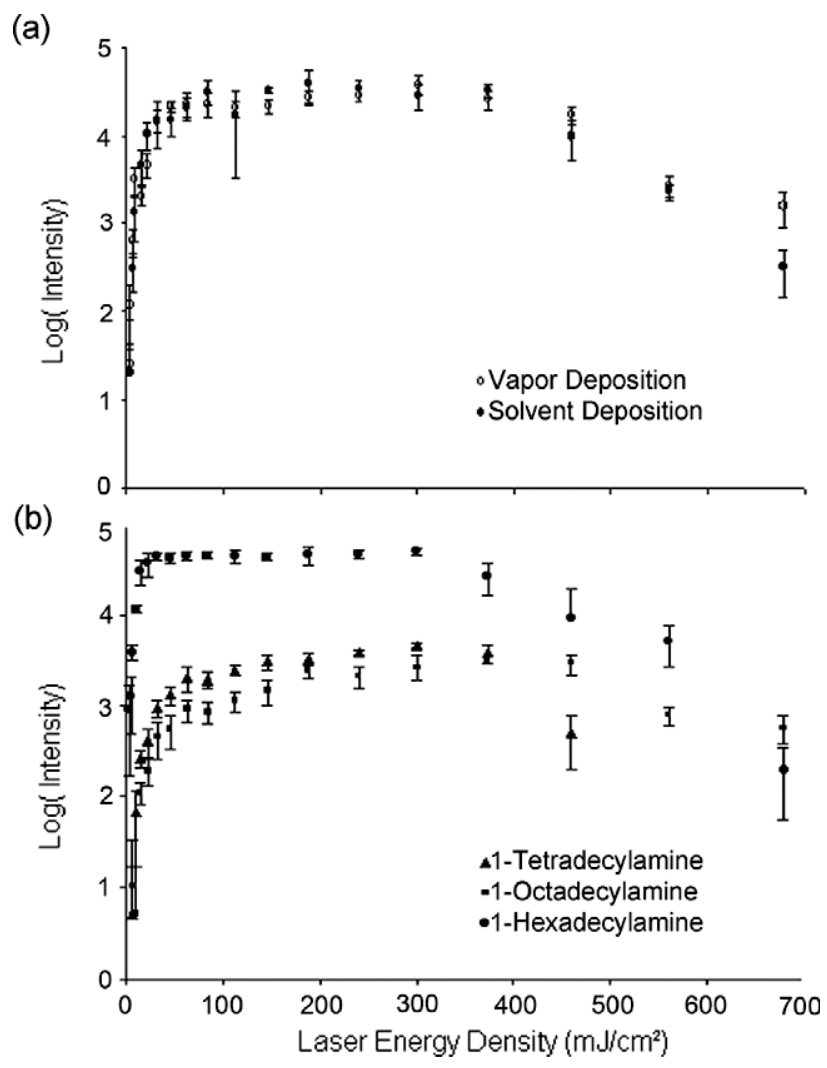

Figure 1. Comparison of the dependence of signal intensity on laser fluence for various sample and depositions: (a) solvent versus vapor deposited 1-hexadecylamine on a vacuum-baked DIOS surface. Solvent deposition of hexadecylamine was drop-coated with a concentration of $1 \mathrm{nmol} / \mu \mathrm{L}$ solution. Similar curves were observed for tetra- and octa-decylamine (not shown). (b) Comparison of laser energy dependence on the DIOS of three aliphatic amines. The laser intensity for the onset of DIOS is observed to be $10 \mathrm{~mJ} / \mathrm{cm}^{2}$ for all three amines. Signals from all three amines have similar laser intensity dependence; initially increasing exponentially, then flattening out, and finally decreasing. Note that signal from the hexadecylamine is saturating the detector, explaining the flattened shape. The higher intensity of the hexadecylamine was probably due to more analyte materials deposited on the surface. Error bars reflect \pm 1 standard deviation (SD) from the mean.

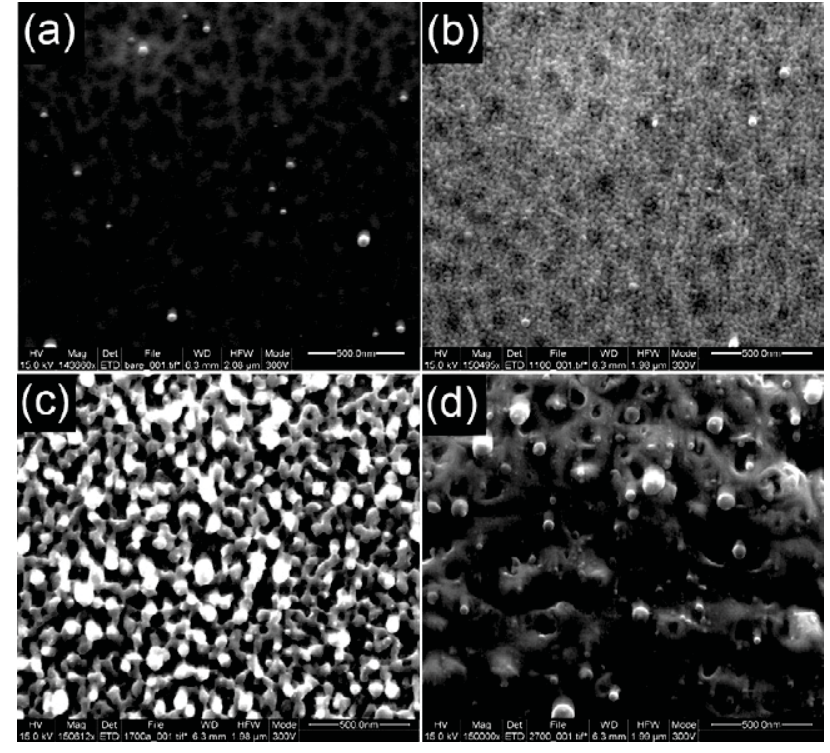

Figure 2. SEM images of DIOS surface exposed to single laser pulses with reveal intensity-dependent surface rearrangement. (a-d) SEM images showing nanostructure irradiated with single laser shot at $337 \mathrm{~nm}$ (scale bar $500 \mathrm{~nm}$ ) (a) surface without irradiation, (b) laser energy of $15 \mathrm{~mJ} / \mathrm{cm}^{2}$ (c) $110 \mathrm{~mJ} / \mathrm{cm}^{2}$, and (d) $970 \mathrm{~mJ} / \mathrm{cm}^{2}$. Native $\mathrm{N}\langle 100\rangle$ silicon etched at $5 \mathrm{~mA}$ for $3 \mathrm{~min}$.

To correlate surface melting and surface ablation, experiments were performed using DIOS surfaces dried at $150{ }^{\circ} \mathrm{C}$ overnight under vacuum. Spectra from single laser shots, integrated over ten positions, were performed with five replicates for each laser setting. This analysis provides additional information of the surface species that is correlated with the changes in surface structure observed in the SEM images (Figure 2). Silicon oxide, $\left(\mathrm{OSiH}^{+}\right)$, which has also been observed using TOF-SIMS (Figure 3a), is observed at laser intensities $>50 \mathrm{~mJ} / \mathrm{cm}^{2}$ and is consistent with the surface restruc-

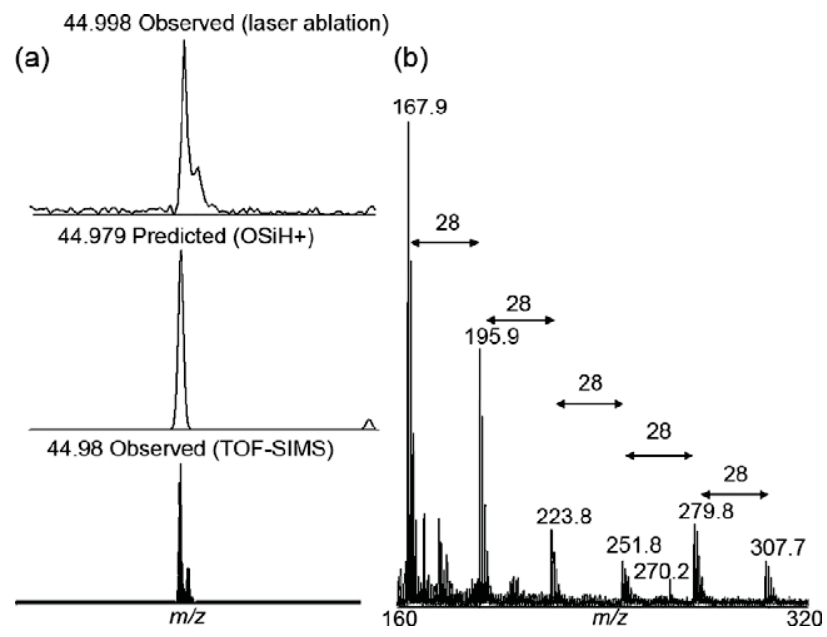

Figure 3. Observed silicon surface ions from DIOS surfaces: (a) $\mathrm{OSiH}^{+}$is observed using laser ablation and TOF-SIMS. (b) DIOS mass spectra showing ablated ions $\left(\mathrm{Si}_{\mathrm{x}}^{+}\right)$off of native $\mathrm{SiH}$ porous silicon surface. Experimental spectra with an averaged of 20 laser shots with $140 \mathrm{~mJ} / \mathrm{cm}^{2}$ per shot. Spectra calibrated using $\mathrm{Na}^{+}$ and $\mathrm{Si}_{10}^{+}$. Note the presence of the octadecylamine analyte at $270 \mathrm{Da}$. 


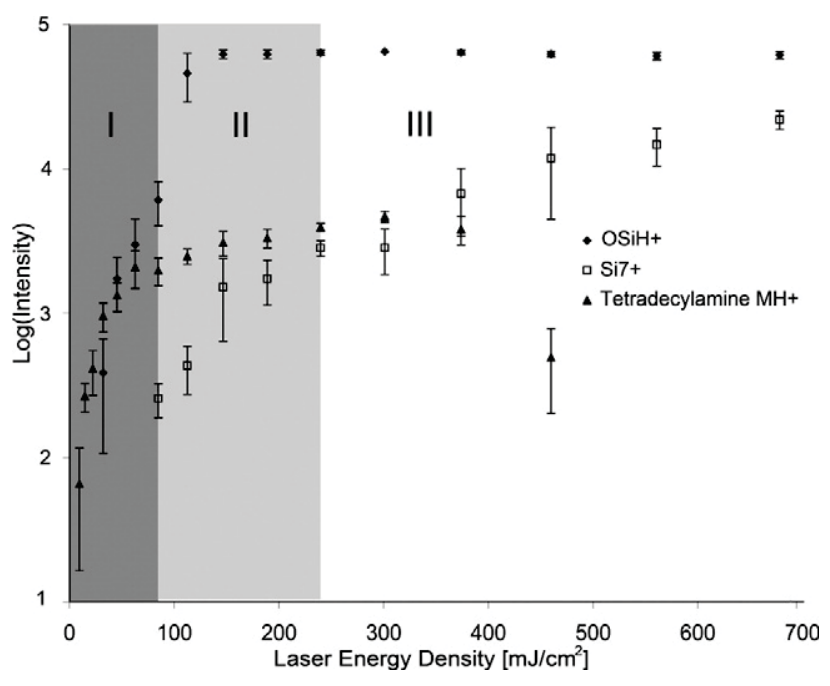

Figure 4. Three laser intensity-dependent regimes of DIOS of vapor-deposited tetraceylamine: Regime I is characterized by strong laser intensity dependence, II by weak but positive laser dependence, and III with negatively laser intensity dependence. These regimes are bounded by the intersections (arrows) with ablated surface ions $\mathrm{OSiH}^{+}$ions and $\mathrm{Si}_{\mathrm{n}}^{+}$(in this case $\mathrm{Si}_{7}^{+}$). Native $\mathrm{N}\langle 100\rangle$ silicon etched at $5 \mathrm{~mA}$ for $3 \mathrm{~min}$. Similar laser energy dependent curves were observed for hexa- and octa-decylamine (not shown). Error bars reflect \pm 1 SD from the mean.

turing to form bulk silicon resulting in the loss of the surface oxide. It is important to note that oxidation is thought to occur by the insertion of oxygen between silicon atoms resulting in $\mathrm{OSiH}$ [18]. At high laser intensities $\left(>100 \mathrm{~mJ} / \mathrm{cm}^{2}\right)$, silicon clusters are observed due to bulk surface melting and ablation (Figures $2 \mathrm{~d}$ and $3 b$ ). It should be noted that surface oxidation with ozone was not found to significantly reduce surface ion detection or DIOS performance, suggesting that some oxidation occurs as part of the etching process or as a result of exposure to atmospheric oxygen.

Figure 4 shows the laser energy dependence of DIOS with vapor-deposited 1-tetradecylamine. There appear to be three regimes of DIOS activity that are correlated with surface melting and ablation: Regime I, a strong dependence on laser intensity as observed between the onset of DIOS $\left(\sim 10 \mathrm{~mJ} / \mathrm{cm}^{2}\right)$ with an initial exponential dependence on laser intensity between 10 and $80 \mathrm{~mJ} /$ $\mathrm{cm}^{2}$; Regime II, a weak but positive dependence on laser intensity $\left(80-250 \mathrm{~mJ} / \mathrm{cm}^{2}\right)$; and finally Regime III, where DIOS decreases with increasing laser intensity $\left(>250 \mathrm{~mJ} / \mathrm{cm}^{2}\right)$. These three regimes are bounded by the intersections with the $\mathrm{OSiH}^{+}$and $\mathrm{Si}_{\mathrm{n}}^{+}$ions as indicated by arrows in Figure 4.

Considering Figures 2 and 4, the three laser intensitydependent regimes support a surface restructuring-driven analyte desorption process: Low laser intensities provide small amounts of energy and results in localized surface changes (Figure 2b, Regime I) triggering desorption of analyte. This highly laser intensity dependent regime suggests that increasing the laser intensity will result in more efficient or larger-scale DIOS. As the laser intensity is increased further (Regime II, Figure 2c), large-scale changes on porous silicon occur, including the loss of the outer surface species (surface oxide). Finally, at high laser intensities (Regime III, Figure 2d), bulk surface destruction and ablation occur, which reduce analyte ions signal. This signal reduction was presumably due to signal suppression from $\mathrm{Si}_{x}^{+}$ions and possibly analyte encapsulation in the melted surface. These observations are consistent with surface reflectivity studies that attributed the large pores (formed at laser intensity $>46 \mathrm{~mJ} / \mathrm{cm}^{2}$ ) to intense hydrogen evaporation from the melting porous silicon and boiling of the silicon [15].

Although the above-mentioned results suggest a surface process, a strictly thermal desorption process is also possible and needs to be considered. For a strictly thermal process, desorption should depend on the heat of vaporization of the adsorbed analyte. Therefore, the threshold desorption fluence of DIOS would vary from molecule to molecule, which is typically not observed (for example, all peptides from a BSA digest are observed at the same threshold desorption fluence). In contrast, a surface restructuring/melting process would be expected to exhibit a threshold (surface rearranging/ melting point). To test this possibility, the DIOS behavior of two other aliphatic amines (hexadecylamine and octadecylamine) are analyzed and compared with the tetradecylamine. Because the molecular structures of these amines are very similar, the $\mathrm{D} / \mathrm{I}$ behavior is expected to be similar. However, these three molecules have dramatically different vapor pressures $(1.89$, $2.90 \times 10^{-1}$, and $4.88 \times 10^{-2} \mathrm{mTorr}$ for tetra-, hexa-, and octa-decylamine, respectively, at room temperature). Therefore, one would expect the three amines to desorb at different laser intensities if DIOS were strictly a thermally driven desorption process. Figure $1 \mathrm{~b}$ reveals that there is a threshold laser intensity onset of DIOS for all three amines at about $10 \mathrm{~mJ} / \mathrm{cm}^{2}$ and all desorption processes show similar laser energy dependence. This observation clearly supports that DIOS is dominated by a surface-driven process, albeit the thermal process cannot be completely ruled out.

\section{Conclusion}

DIOS signal, surface restructuring, and surface melting are found to depend on laser fluence. The correlation between the signal and changes in surface morphology suggests that the high surface area of porous silicon drives the desorption of analyte and the hydrogen content of the surface provides a source of protons for ionization. The DIOS process is found to be highly laser energy dependent and correlated with the appearance of surface ions $\left(\mathrm{Si}_{\mathrm{n}}^{+}\right.$and $\left.\mathrm{OSiH}^{+}\right)$. A threshold laser intensity for molecular ion formation is observed (10 $\mathrm{mJ} / \mathrm{cm}^{2}$ ), even with molecules having different vapor pressures, supporting a view that DIOS is driven by surface restructuring and not a strictly thermal process. The DIOS performance can be further improved by use 
of high surface area silicon substrates such as silicon nanowires [7].

\section{Acknowledgments}

The authors gratefully acknowledge support from Department of Energy Grant MAGGIE DE-AC02-05CH11231 and National Institute of Mental Health Grant SNAPS 5 P30 MH-062261-06. They also thank Dr. Kuang Jen Wu and Ligang Wu for useful discussions and TOF-SIMS surface characterization.

\section{References}

1. Wei, J.; Buriak, J. M.; Siuzdak, G. Desorption-Ionization Mass Spectrometry on Porous Silicon. Nature 1999, 399, 243-246.

2. Karas, M.; Bahr, U.; Ingendoh, A.; Nordhoff, E.; Stahl, B.; Strupat, K.: Hillenkamp, F. Principles and Applications of Matrix-Assisted UV Laser Desorption Ionization Mass-Spectrometry. Anal. Chim. Acta 1990, 241, 175-185.

3. Karas, M.; Hillenkamp, F. Laser Desorption Ionization of Proteins with Molecular Masses Exceeding 10,000 Daltons. Anal. Chem. 1988, 60, 2299-2301.

4. Pachuta, S. J.; Cooks, R. G. Mechanisms in Molecular Sims. Chem. Rev. $1987,87,647-669$

5. Kruse, R. A.; Li, X. L.; Bohn, P. W.; Sweedler, J. V. Experimental Factors Controlling Analyte Ion Generation in Laser Desorption/Ionization Mass Spectrometry on Porous Silicon. Anal. Chem. 2001, 73, 3639-3645.

6. Ren, S. F.; Zhang, L.; Cheng, Z. H.; Guo, Y. L. Immobilized Carbon Nanotubes as Matrix for MALDI-TOF-MS Analysis: Applications to Neutral Small Carbohydrates. J. Am. Soc. Mass Spectrom. 2005, 16, 333-339.

7. Go, E. P.; Apon, J. V.; Luo, G.; Saghatelian, A.; Daniels, R. H.; Sahi, V.; Dubrow, R.; Cravatt, B. F.; Vertes, A.; Siuzdak, G. Desorption/ Ionization on Silicon Nanowires. Anal. Chem. 2005, 77, 1641-1646.
8. Luo, G. H.; Chen, Y.; Daniels, H.; Dubrow, R.; Vertes, A. Internal Energy Transfer in Laser Desorption/Ionization from Silicon Nanowires. J. Phys. Chem. B 2006, 110,13381-13386.

9. Okuno, S.; Arakawa, R.; Okamoto, K.; Matsui, Y.; Seki, S.; Kozawa, T.; Tagawa, S.; Wada, Y. Requirements for Laser-induced Desorption/ Ionization on Submicrometer Structures. Anal. Chem. 2005, 77, 5364 5369.

10. Lewis, W. G.; Shen, Z. X.; Finn, M. G.; Siuzdak, G. Desorption/ Ionization on Silicon (DIOS) Mass Spectrometry: Background and Applications. Int. J. Mass Spectrom. 2003, 226, 107-116.

11. Trauger, S. A.; Go, E. P.; Shen, Z. X.; Apon, J. V.; Compton, B. J.; Bouvier, E. S. P.; Finn, M. G.; Siuzdak, G. High Sensitivity and Analyte Capture with Desorption/Ionization Mass Spectrometry on Silylated Porous Silicon. Anal. Chem. 2004, 76, 4484-4489.

12. Peterson, D. S. Matrix-free Methods for Laser Desorption/Ionization Mass Spectrometry. Mass Spectrom. Rev. 2007, 26, 19-34.

13. Herino, R.; Perio, A.; Bomchil, G. Microstructure of Porous Silicon and Its Evolution with Temperature. Mater. Lett. 1984, 2, 519-523.

14. Broughton, J. Q.; Li, X. P. Phase Diagram of Silicon by Molecular Dynamics. Phys. Rev. B 1987, 35, 9120-9127.

15. Timoshenko, V. Y.; Dittrich, T.; Sieber, I.; Rappich, J.; Kamenev, B. V.; Kashkarov, P. K. Laser-induced Melting of Porous Silicon. Physica Status Solidi A Appl. Res. 2000, 182, 325-330.

16. Luo, G. H.; Chen, Y.; Siuzdak, G.; Vertes, A. Surface Modification and Laser Pulse Length Effects on Internal Energy Transfer in DIOS. J. Phys. Chem. B 2005, 109, 24450-24456.

17. Nakajima, A.; Itakura, T.; Watanabe, S.; Nakayama, N. Photoluminescence of Porous Si, Oxidized Then Deoxidized Chemically. Appl. Phys. Lett. 1992, 61, 46-48.

18. Collins, R. T.; Tischler, M. A.; Stathis, J. H. Photoinduced Hydrogen Loss from Porous Silicon. Appl. Phys. Lett. 1992, 61, 1649-1651.

19. Pusel, A.; Wetterauer, U.; Hess, P. Photochemical Hydrogen Desorption from H-terminated Silicon(111) by VUV Photons. Phys. Rev. Lett. 1998, 81, 645-648.

20. Hamilton, B. Porous Silicon. Semiconduct. Sci. Technol. 1995, 10, 11871207

21. Shen, Z.; Thomas, J. J.; Averbuj, C.; Broo, K. M.; Engelhard, M.; Crowell, J. E.; Finn, M. G.; Siuzdak, G. Porous Silicon as a Versatile Platform for Laser Desorption/Ionization Mass Spectrometry. Anal. Chem. 2001, 73, 612-619. 